\title{
The Shilnikov type homoclinic orbits of perturbed cubic polynomial Moon-Rand systems
}

\author{
Dandan Xie ${ }^{a, b}$, Yinlai Jin ${ }^{a, *}$, Feng $\mathrm{Li}^{\mathrm{a}}$, Nana Zhang ${ }^{\mathrm{a}, \mathrm{b}}$ \\ a School of Mathematics and Statistics, Linyi University, Linyi, Shandong, 276005, P. R. China. \\ ${ }^{b}$ School of Mathematics and Statistics, Shandong Normal University, Jinan, Shandong, 250014, P. R. China.
}

\begin{abstract}
In this paper, perturbed polynomial Moon-Rand systems are considered. The Padé approximant and analytic solution in the neighborhood of the initial value are introduced into the process of constructing the Shilnikov type homoclinic orbits for three dimensional nonlinear dynamical systems. In order to get real bifurcation parameters, four undetermined coefficients are introduced including three initial values about position and the value of bifurcation parameter. By the eigenvectors of its all eigenvalues, the value of the bifurcation parameter and three initial values about position are obtained directly. And, the analytical expressions of the Shilnikov type homoclinic orbits are achieved and the deletion errors relative to the practical system are given. In the end, we roughly predict when the horseshoe chaos occurs. (c)2017 All rights reserved.
\end{abstract}

Keywords: Padé approximant, analytic solution, Shilnikov theorem, homoclinic orbit.

2010 MSC: 34C23, 34C37, 37C29.

\section{Introduction}

Bifurcations and chaos of three dimensional (3D) systems have been becoming the core research content of the complex dynamical behavior in nonlinear systems. The Padé approximant was successfully used for the homoclinic orbit of two dimensional axisymmetric breather system by Emaci et al. [1]. The Padé approximation was extended to generate the solutions which satisfy the boundary value of homoclinic and heteroclinic solutions, and then to derive the critical values of bifurcation in the corresponding conservative, autonomous and nonautonomous systems with quadratic and cubic nonlinear oscillators, and the system parameters of the linear and nonlinear terms were variable [2,3]. Quasi-Padé approximant was used to construct the homoclinic orbit for a nonlinear Schrödinger equation system by Manucharyan and Mikhlin [6]. Analytic homoclinic orbit in the nonautonomous Duffing equation and the Van Der Pol-Duffing equation with weakly coupled nonlinear oscillators was computed by Mikhlin [7]. Moreover, Feng et al. constructed the homoclinic and heteroclinic orbits in general asymmetric double well and triple well systems to improve the accuracy of the threshold for the onset of chaos [3].

In our research, the Shilnikov homoclinic and heteroclinic theorems are important analytical theorems for chaotic motion in 3D nonlinear dynamical systems. The specific analytical expressions of the Shilnikov

\footnotetext{
${ }^{*}$ Corresponding author

Email addresses: xiedandan01@sina.com (Dandan Xie), jinyinlai@sina.com (Yinlai Jin), lf0539@126.com (Feng Li), zhangnana01@sina.com (Nana Zhang)
}

doi:10.22436/jmcs.017.03.06 
type homoclinic orbits are rarely given. Mikhlin used a quasi-Padé approximant method to construct homoclinic orbit of the Lorenz equation [8]. Vakakis and Azeez expressed the solution in power series such as $t \rightarrow \pm 0$ and introduced global approximant to match the local solutions by means of a Padé-like procedure [9]. According to the characteristics of the $n$-dimensional system, Li and Zhu constructed the s-dimensional series expression of the stable and unstable manifolds, which formed a homoclinic orbit near the hyperbolic singular point of the Lorenz system [5]. For [10], the bifurcate parameter value was selected in a reasonable region at the requirement of the Shilnikov theorem, then the analytic expression of the Shilnikov type homoclinic orbit was accomplished by the series expression of manifold. In [4], as the global approximant were analytically expressed in a series, so they can be expanded in power series in the neighborhood of $t=0$. And, in [4], the initial conditions of the orbit satisfy $x(0)=x_{0}, y(0)=y_{0}$, and $\dot{z}(0)=0$, so, the values of bifurcation parameter and initial value $x_{0}$, yo were obtained directly.

In this paper, we study the perturbed cubic polynomial Moon-Rand system. By using the same method we get the bifurcate parameter value which is complex number. This is not what we want to get. Here, we present a method of how to find homoclinic orbit and bifurcation parameter which is real value in a 3D autonomous nonlinear system, and, the homoclinic orbit is Shilnikov type homoclinic orbit. The initial conditions of the orbit in [4] are modified as $x(0)=x_{0}, y(0)=y_{0}, z(0)=z_{0}$. The Padé approximation method is introduced and the analytic solution can be expanded in power series in the neighbourhood of $t=0$. Thus, the values of bifurcation parameter and initial values of $x_{0}, y_{0}, z_{0}$ are obtained directly. The analytical expressions of the Shilnikov type homoclinic orbit are also formed. The deletion error relative to the practical system is achieved by programming.

The rest of this paper is organized as follows. In Section 2, Shilnikov theorem and in Section 3, eigenvalues analysis of the system are presented. In Section 4, the calculation procedures of the presented method in this paper are introduced. The final section, i.e., Section 5, is devoted to some conclusions that we got from the present study.

\section{Shilnikov theorem}

In this section, we present Shilnikov theorem. Shilnikov proved that under certain conditions a saddlefoci homoclinic orbit in its neighborhood has a countable set of periodic trajectories, which can lead to the formation of chaotic attractor. For chaos to occur within a three dimensional phase space, it requires the system

$$
\frac{d x}{d t}=f(x), t \in R, x \in R^{3},
$$

where the vector field $f: R^{3} \rightarrow R^{3}$ is $p$-times differentiable $(p \geqslant 1)$ with a continuous derivative (so called $C^{p}$ ), and $R^{3}$ stands for the real three dimensional space. We call the equilibrium point $x_{0} \in R^{3}$, a hyperbolic saddle-foci, if the eigenvalues of the $3 \times 3$ real matrix $A=\operatorname{Df}\left(x_{0}\right)$, the Jacobin derivative of $f$ at $x_{0}$, are of the form

$$
\lambda_{1}=r, \lambda_{2,3}=p \pm i q, r p<0, q \neq 0,
$$

where $r, p, q$ are real. The Shilnikov theorem will be described as follows:

Theorem 2.1 (Shilnikov theorem, [10]). For Eq. (2.1), suppose that $x_{0}$ is a hyperbolic saddle-foci equilibrium point, the corresponding Jacobin matrix $\operatorname{Df}\left(x_{0}\right)$ has eigenvalues $\lambda_{1}=r, \lambda_{2,3}=p \pm i q$, which satisfy $r<0, p>0$, $q \neq 0,|r|>p$, and there exists a homoclinic orbit based at $x_{0}$. Then, system (2.1) exhibits horseshoe chaos.

\section{Eigenvalues analysis of the system}

We consider a three dimensional system

$$
\left\{\begin{array}{l}
\dot{x}=\alpha x+y, \\
\dot{y}=-x+\alpha y-x z \\
\dot{z}=-\lambda z+C_{30} x^{3}+C_{21} x^{2} y+C_{12} x y^{2}+C_{03} y^{3},
\end{array}\right.
$$

which is formally evolved from the three dimensional system 


$$
\left\{\begin{array}{l}
\dot{x}=y \\
\dot{y}=-x-x z \\
\dot{z}=-\lambda z+C_{30} x^{3}+C_{21} x^{2} y+C_{12} x y^{2}+C_{03} y^{3}
\end{array}\right.
$$

with the primary target of promoting or eliminating chaos. Here we discuss the Jacobin matrix

$$
A=\left[\begin{array}{ccc}
\alpha & 1 & 0 \\
-1 & \alpha & 0 \\
0 & 0 & -\lambda
\end{array}\right]
$$

at the equilibrium point $(0,0,0)$. The characteristic polynomial is

$$
k^{3}+(\lambda-2 \alpha) k^{2}+\left(\alpha^{2}-2 \alpha \lambda+1\right) k+\alpha^{2} \lambda+\lambda=0 .
$$

By (3.2), we get

$$
k_{1}=-\lambda, k_{2}=\alpha-i, k_{3}=\alpha+i .
$$

Based on Shilnikov theorem, we must get a negative real root and a pair of conjugate complex roots with positive real parts. So $\lambda>0, \alpha>0$.

According to Shilnikov theorem, when $\lambda>\alpha>0$, (3.1) may has a homoclinic orbit connecting the equilibrium point $(0,0,0)$. It is our intention to find the approximate expression of homoclinic orbit based at $x_{0}$ of the system (3.3).

In this paper, let the initial values are $x_{0}, y_{0}, z_{0}, \alpha$, in which $\alpha$ is the bifurcation parameter, other parameters are chosen to be

$$
\lambda=5, C_{30}=1, C_{21}=1, C_{12}=1, C_{03}=1
$$

Thus, (3.1) turns to

$$
\left\{\begin{array}{l}
\dot{x}=\alpha x+y \\
\dot{y}=-x+\alpha y-x z \\
\dot{z}=-5 z+x^{3}+x^{2} y+x y^{2}+y^{3} .
\end{array}\right.
$$

The Shilnikov sense Smale horseshoe chaos is predicted to occur in a range of $5>\alpha>0$ in terms of the Shilnikov theorem.

\section{Finding analytic homoclinic solution}

Since the calculation process is complicated and time-consuming in many systems when using the invariant manifold theorem to calculate the stable and unstable manifold equation, series expressions of the stable and unstable manifolds at a hyperbolic saddle-focus point are introduced by taking the 1D manifold and 2D manifold into consideration in calculation. The 1D manifold in (3.3) can be expressed as

$$
x^{[+]}(t)=\sum_{k=0}^{n} a_{k} e^{k k_{1} t}, y^{[+]}(t)=\sum_{k=0}^{n} b_{k} e^{k k_{1} t}, z^{[+]}(t)=\sum_{k=0}^{n} c_{k} e^{k k_{1} t},(t \rightarrow+\infty),
$$

where $a_{k}, b_{k}$, and $c_{k}$ are undetermined coefficients.

Similarly, the 2D manifold in (3.3) can be expressed as

$$
\begin{aligned}
x^{[-]}(t)=\sum_{k=0}^{n} \sum_{j=0}^{n-k} a_{k, j} e^{k k_{2} t+j k_{3} t}, y^{[-]}(t) & =\sum_{k=0}^{n} \sum_{j=0}^{n-k} b_{k, j} e^{k k_{2} t+j k_{3} t}, \\
z^{[-]}(t) & =\sum_{k=0}^{n} \sum_{j=0}^{n-k} c_{k, j} e^{k k_{2} t+j k_{3} t},(t \rightarrow-\infty),
\end{aligned}
$$


where $a_{k, j}, b_{k, j}$, and $c_{k, j}$ are undetermined coefficients as before.

Here, analytic solutions in the neighborhood of the initial value are considered to eliminate the disjoint problem between 1D and 2D manifold expressions in traditional algorithms. The relations between 1D and 2D manifold expressions are strengthened. Since the homoclinic solution is analytic in the neighborhood of $t=0$, the solution in power series as $t \rightarrow \pm 0$ can be expressed as follows

$$
x^{[0]}(t)=\sum_{k=0} x_{k}^{[0]} t^{k}, y^{[0]}(t)=\sum_{k=0} x_{k}^{[0]} t^{k}, z^{[0]}(t)=\sum_{k=0} x_{k}^{[0]} t^{k}, \quad(t \rightarrow \pm \infty) .
$$

Substituting (4.3) into (3.3) and matching coefficients of respective powers of $t$, we obtain the recursive expressions represented by constant coefficients of series as follows

$$
\begin{aligned}
x_{k+1}^{[0]}= & \frac{1}{k+1}\left[\alpha x_{k}^{[0]}+y_{k}^{[0]}\right], \\
y_{k+1}^{[0]}= & \frac{1}{k+1}\left[-x_{k}^{[0]}+\alpha y_{k}^{[0]}-\sum_{j=0}^{k} x_{j}^{[0]} z_{k-j}^{[0]}\right], \\
z_{k+1}^{[0]}= & \frac{1}{k+1}\left[-5 z_{k}^{[0]}+\sum_{j=0}^{k} \sum_{h=0}^{k-j} x_{j}^{[0]} x_{h}^{[0]} x_{k-j-h}^{[0]}+\sum_{j=0}^{k} \sum_{h=0}^{k-j} x_{j}^{[0]} x_{h}^{[0]} y_{k-j-h}^{[0]}\right. \\
& \left.+\sum_{j=0}^{k} \sum_{h=0}^{k-j} x_{j}^{[0]} y_{h}^{[0]} y_{k-j-h}^{[0]}+\sum_{j=0}^{k} \sum_{h=0}^{k-j} y_{j}^{[0]} y_{h}^{[0]} y_{k-j-h}^{[0]}\right] .
\end{aligned}
$$

Since system (3.3) is autonomous, the initial conditions of the orbit can arbitrarily be at the point where $x(0)=x_{0}=a, y(0)=y_{0}=b, z(0)=z_{0}=c$. Then,

$$
x_{0}^{[0]}=x_{0}=a, y_{0}^{[0]}=y_{0}=b, z_{0}^{[0]}=z_{0}=c,
$$

where $x_{0}, y_{0}, z_{0}$ are undetermined initial values. As $t \rightarrow \pm 0$, the positive and negative approximants $x^{[+]}(t)$ and $x^{[-]}(t)$ are expanded in power series and equated to the corresponding local homoclinic expansion $x^{[0]}(t)$, that is $x^{[ \pm]}(t) \approx x^{[0]}(t)$. Then we can get

$$
x^{[+]}(t)=\sum_{k=0}^{n} a_{k} e^{k k_{1} t}=a_{0}+a_{1} e^{k_{1} t}+a_{2} e^{2 k_{1} t}+\cdots+a_{n} e^{n k_{1} t} \approx x_{0}^{[0]}+x_{1}^{[0]} t+x_{2}^{[0]} t^{2}+\cdots
$$

Matching the coefficients of respective powers of $t$, the coefficients $a_{1}, \cdots, a_{n}$ are evaluated in terms of $x_{i}^{[0]}$ as follows

$$
\left\{\begin{array}{c}
a_{1} \\
a_{2} \\
\cdots \\
a_{n}
\end{array}\right\}=\left[\begin{array}{cccc}
1 & 1 & \ldots & 1 \\
k_{1} & 2 k_{1} & \ldots & n k_{1} \\
\ldots & \ldots & & \ldots \\
k_{1}^{n-1} & \left(2 k_{1}\right)^{n-1} & \ldots & \left(n k_{1}\right)^{n-1}
\end{array}\right]^{-1} \times\left\{\begin{array}{c}
x_{0}^{[0]} \\
1 ! x_{1}^{[0]} \\
\ldots \\
(n-1) ! x_{n-1}^{[0]}
\end{array}\right\}
$$

Similarly, the 2D manifold equation is obtained as

$$
\begin{aligned}
x^{[-]}(t)= & \sum_{k=0}^{n} \sum_{j=0}^{n-k} a_{k, j} e^{k k_{2} t+j k_{3} t} \\
= & a_{0,0}+a_{1,0} e^{k_{2} t}+a_{2,0} e^{2 k_{2} t}+\cdots+a_{n, 0} e^{n k_{2} t}+a_{0,1} e^{k_{3} t}+a_{0,2} e^{2 k_{3} t}+\cdots+a_{0, n} e^{n k_{3} t} \\
& +a_{1,1} e^{k_{2} t+k_{3} t}+a_{1,2} e^{k_{2} t+2 k_{3} t}+\cdots+a_{1, n-1} e^{k_{2} t+(n-1) k_{3} t} \\
& +a_{2,1} e^{2 k_{2} t+k_{3} t}+\cdots+a_{n-1,1} e^{(n-1) k_{2} t+k_{3} t}+\cdots \approx x_{0}^{[0]}+x_{1}^{[0]} t+x_{2}^{[0]} t^{2}+\cdots
\end{aligned}
$$


The above equation can be simplified into

$$
\left\{\begin{array}{c}
a_{1,0} \\
a_{2,0} \\
\vdots \\
a_{n, 0} \\
a_{0,1} \\
a_{0,2} \\
\vdots \\
a_{0, n} \\
a_{1,1} \\
a_{1,2} \\
\vdots \\
a_{1, n-1} \\
a_{2,1} \\
\vdots \\
a_{n-1,1}
\end{array}\right\}_{s \times 1}=\left[\begin{array}{lll}
A & B & C
\end{array}\right]_{s \times s}^{-1} \times\left\{\begin{array}{c}
x_{0}^{[0]} \\
1 ! x_{1}^{[0]} \\
\cdots \\
(s-1) ! x_{s-1}^{[0]}
\end{array}\right\}_{s \times 1}
$$

where $s=\frac{n(n+3)}{2}$,

$$
\begin{aligned}
& A=\left[\begin{array}{cccc}
1 & 1 & \ldots & 1 \\
k_{2} & 2 k_{2} & \ldots & n k_{2} \\
\ldots & \ldots & & \ldots \\
k_{2}^{s-1} & \left(2 k_{2}\right)^{s-1} & \ldots & \left(n k_{2}\right)^{s-1}
\end{array}\right]_{s \times n} \quad, \quad B=\left[\begin{array}{cccc}
1 & 1 & \ldots & 1 \\
k_{3} & 2 k_{3} & \ldots & n k_{3} \\
\ldots & \ldots & & \ldots \\
k_{3}^{s-1} & \left(2 k_{3}\right)^{s-1} & \ldots & (n k)^{s-1}
\end{array}\right]_{s \times n} \\
& \mathrm{C}=\left[\begin{array}{ccccccc}
1 & 1 & \ldots & \ldots & \ldots & \ldots & 1 \\
k_{2}+k_{3} & k_{2}+2 k_{3} & \ldots & k_{2}+(n-1) k_{3} & 2 k_{2}+k_{3} & \ldots & (n-1) k_{2}+k_{3} \\
\ldots & \ldots & \ldots & \ldots & \ldots & \ldots & \ldots \\
\left(k_{2}+k_{3}\right)^{s-1} & \left(k_{2}+2 k_{3}\right)^{s-1} & \ldots & \left(k_{2}+(n-1) k_{3}\right)^{s-1} & \left(2 k_{2}+k_{3}\right)^{s-1} & \ldots & \left((n-1) k_{2}+k_{3}\right)^{s-1}
\end{array}\right]_{s \times \frac{n(n-1)}{2}} . \\
& \text { Similarly, }\left\{\begin{array}{c}
b_{1} \\
b_{2} \\
\vdots \\
b_{n}
\end{array}\right\},\left\{\begin{array}{c}
c_{1} \\
c_{2} \\
\vdots \\
c_{n}
\end{array}\right\},\left\{\begin{array}{c}
b_{1,0} \\
b_{2,0} \\
\vdots \\
b_{n, 0} \\
b_{0,1} \\
b_{0,2} \\
\vdots \\
b_{0, n} \\
b_{1,1} \\
b_{1,2} \\
\vdots \\
b_{1, n-1} \\
b_{2,1} \\
\vdots \\
b_{n-1,1}
\end{array}\right\}_{s \times 1},\left\{\begin{array}{c}
c_{1,0} \\
c_{2,0} \\
\vdots \\
c_{n, 0} \\
c_{0,1} \\
c_{0,2} \\
\vdots \\
c_{0, n} \\
c_{1,1} \\
c_{1,2} \\
\vdots \\
c_{1, n-1} \\
c_{2,1} \\
\vdots \\
c_{n-1,1}
\end{array}\right\}_{s \times 1} \text { are evaluated in terms of } y_{i}^{[0]}, z_{i}^{[0]} \text {. }
\end{aligned}
$$

Here, $a_{0}, b_{0}, c_{0}$ and $a_{0,0}, b_{0,0}, c_{0,0}$ are the component values of system at the singular point, namely zero [10]. And, approximant with superscript $+(-)$ are termed positive (negative) approximant and are valid only for positive (negative) values of $t$. The order of the exponents appearing in the positive and 
negative approximants are identical to those of the asymptotic expansions in (4.1) and (4.2) for $t>0$ and $\mathrm{t}<0$, respectively.

Because $k_{1}, k_{2}, k_{3}$ are eigenvalues of linear part of system, so bringing (4.1) and (4.2) back to the original equation, we can easily obtain

$$
\left(a_{1}, b_{1}, c_{1}\right)=\mu_{1}\left(v_{1,1}, v_{1,2}, v_{1,3}\right), \mu_{1} \in\left[-\mu_{1 m}, \mu_{1 m}\right]
$$

and

$$
\begin{aligned}
& \left(a_{1,0}, b_{1,0}, c_{1,0}\right)=\mu_{2}\left(v_{2,1}, v_{2,2}, v_{2,3}\right), \mu_{2} \in\left[-\mu_{2 m}, \mu_{2 m}\right], \\
& \left(a_{0,1}, b_{0,1}, c_{0,1}\right)=\mu_{3}\left(v_{3,1}, v_{3,2}, v_{3,3}\right), \mu_{3} \in\left[-\mu_{3 m}, \mu_{3 m}\right],
\end{aligned}
$$

where $\mu_{1 \mathrm{~m}}, \mu_{2 \mathrm{~m}}, \mu_{3 \mathrm{~m}}$ are the maximum constants to keep series expressions (4.1) and (4.2) convergent, $\left(v_{1,1}, v_{1,2}, v_{1,3}\right)$ is the eigenvector of eigenvalue $k_{1},\left(v_{2,1}, v_{2,2}, v_{2,3}\right)$ is the eigenvector of eigenvalue $k_{2}$, and $\left(v_{3,1}, v_{3,2}, v_{3,3}\right)$ is the eigenvector of eigenvalue $k_{3}([5])$.

Considering (4.7), (4.8), and (4.9), it is easy to verify that (4.8) is equivalent to (4.9), so we only need (4.7) and (4.8), or (4.7) and (4.9) are true. Moreover, we know that if one of $v_{1,1}, v_{1,2}, v_{1,3}, v_{2,1}, v_{2,2}, v_{2,3}$, $v_{3,1}, v_{3,2}, v_{3,3}$ is zero, then, we must get one of $a_{1}, b_{1}, c_{1}, a_{1,0}, b_{1,0}, c_{1,0}, a_{0,1}, b_{0,1}, c_{0,1}$ is zero. So without loss of generality, linear part of general $3 \mathrm{D}$ autonomous system is

$$
\left[\begin{array}{ccc}
\lambda_{1} & 0 & 0 \\
0 & \alpha & -w \\
0 & w & \alpha
\end{array}\right]
$$

where $\lambda_{1} \in \mathrm{R}, \lambda_{2}=\alpha+\mathrm{i} w, \lambda_{3}=\alpha-\mathrm{i} w, \alpha \in \mathrm{R}, w \in \mathrm{R}$.

Now, again we consider the system

$$
\left\{\begin{array}{l}
\dot{x}=\alpha x+y \\
\dot{y}=-x+\alpha y-x z \\
\dot{z}=-5 z+x^{3}+x^{2} y+x y^{2}+y^{3}
\end{array}\right.
$$

Taking the third-order approximant of (4.1), the powers are $k_{1}$-th, $2 k_{1}$-th and $3 k_{1}$-th for the 1D equation, taking the second-order approximant of Eq. (4.2), the powers are $k_{2}-t h, 2 k_{2}-t h, k_{3}-t h, 2 k_{3}-t h, k_{2}+k_{3}$-th for the $2 \mathrm{D}$ equation. And, the selected initial conditions of the orbit can arbitrarily be at the point where $x(0)=x_{0}=a, y(0)=y_{0}=b, z(0)=z_{0}=c$.

Using (4.4), computing by programming we can easily get $\left\{\begin{array}{c}x_{0}^{[0]} \\ x_{1}^{[0]} \\ x_{2}^{[0]} \\ x_{3}^{[0]} \\ x_{4}^{[0]}\end{array}\right\},\left\{\begin{array}{c}y_{0}^{[0]} \\ y_{1}^{[0]} \\ y_{2}^{[0]} \\ y_{3}^{[0]} \\ y_{4}^{[0]}\end{array}\right\},\left\{\begin{array}{c}z_{0}^{[0]} \\ z_{1}^{[0]} \\ z_{2}^{[0]} \\ z_{3}^{[0]} \\ z_{4}^{[0]}\end{array}\right\}$. Then, by

(4.5) and (4.6), we can immediately get $\left\{\begin{array}{l}a_{1} \\ a_{2} \\ a_{3}\end{array}\right\},\left\{\begin{array}{l}b_{1} \\ b_{2} \\ b_{3}\end{array}\right\},\left\{\begin{array}{l}c_{1} \\ c_{2} \\ c_{3}\end{array}\right\},\left\{\begin{array}{l}a_{1,0} \\ a_{2,0} \\ a_{0,1} \\ a_{0,2} \\ a_{1,1}\end{array}\right\},\left\{\begin{array}{l}b_{1,0} \\ b_{2,0} \\ b_{0,1} \\ b_{0,2} \\ b_{1,1}\end{array}\right\},\left\{\begin{array}{l}c_{1,0} \\ c_{2,0} \\ c_{0,1} \\ c_{0,2} \\ c_{1,1}\end{array}\right\}$.

Moreover, we also know they are all functions of $a, b, c, \alpha$. So, if we want to get 1D and 2D manifold expressions, we must determine $a, b, c, \alpha$. Next, making full use of the eigenvector $\left(v_{i, 1}, v_{i, 2}, v_{i, 3}\right)$ of eigenvalue $k_{i}$, we can get a simultaneous equation. By computing, the Jacobian matrices of the system (3.3) have eigenvalue $k_{1}=-5$ corresponding to eigenvector $(0,0,1)$, eigenvalue $k_{2}=-i+\alpha$ corresponding to eigenvector $(1,-i, 0)$, eigenvalue $k_{3}=i+\alpha$ corresponding to eigenvector $(1, i, 0)$. 
Using conclusion (4.7) and (4.8), we easily get a simultaneous equation

$$
\left\{\begin{array}{l}
a_{1}=0 \\
b_{1}=0 \\
\operatorname{Re}\left(c_{1,0}\right)=0 \\
\operatorname{Im}\left(c_{1,0}\right)=0
\end{array}\right.
$$

meanwhile,

$$
\frac{\mathrm{a}_{1,0}}{\mathrm{~b}_{1,0}}=\mathrm{i}
$$

also must be satisfied as soon as possible, where $a_{1}, b_{1}, a_{1,0}, b_{1,0}, c_{1,0}$ are functions of $a, b, c, \alpha$.

From (4.10) and (4.11), we can directly obtain the values of $a, b, c, \alpha$. Then, $\left\{\begin{array}{l}a_{1} \\ a_{2} \\ a_{3}\end{array}\right\},\left\{\begin{array}{l}b_{1} \\ b_{2} \\ b_{3}\end{array}\right\}$, $\left\{\begin{array}{l}c_{1} \\ c_{2} \\ c_{3}\end{array}\right\},\left\{\begin{array}{l}a_{1,0} \\ a_{2,0} \\ a_{0,1} \\ a_{0,2} \\ a_{1,1}\end{array}\right\},\left\{\begin{array}{l}b_{1,0} \\ b_{2,0} \\ b_{0,1} \\ b_{0,2} \\ b_{1,1}\end{array}\right\},\left\{\begin{array}{l}c_{1,0} \\ c_{2,0} \\ c_{0,1} \\ c_{0,2} \\ c_{1,1}\end{array}\right\}$ are known. Because the coefficients include complex number, the homoclinic solution in positive and negative directions can be written in the following form

$$
\begin{aligned}
& x^{[+]}(t)=a_{1} e^{k_{1} t}+a_{2} e^{2 k_{1} t}+a_{3} e^{3 k_{1} t}, \\
& y^{[+]}(t)=b_{1} e^{k_{1} t}+b_{2} e^{2 k_{1} t}+b_{3} e^{3 k_{1} t}, \\
& z^{[+]}(t)=c_{1} e^{k_{1} t}+c_{2} e^{2 k_{1} t}+c_{3} e^{3 k_{1} t}, \\
& x^{[-]}(t)=\operatorname{Re}\left(a_{1,0} e^{k_{2} t}+a_{2,0} e^{2 k_{2} t}+a_{0,1} e^{k_{3} t}+a_{0,2} e^{2 k_{3} t}+a_{1,1} e^{k_{2} t+k_{3} t}\right), \\
& y^{[-]}(t)=\operatorname{Re}\left(b_{1,0} e^{k_{2} t}+b_{2,0} e^{2 k_{2} t}+b_{0,1} e^{k_{3} t}+b_{0,2} e^{2 k_{3} t}+b_{1,1} e^{k_{2} t+k_{3} t}\right), \\
& z^{[-]}(t)=\operatorname{Re}\left(c_{1,0} e^{k_{2} t}+c_{2,0} e^{2 k_{2} t}+c_{0,1} e^{k_{3} t}+c_{0,2} e^{2 k_{3} t}+c_{1,1} e^{k_{2} t+k_{3} t}\right) .
\end{aligned}
$$

The above calculation of homoclinic orbit can be achieved by programming. That is the analytic homoclinic solution can be obtained.

Solving equation (4.10)-(4.11) by MATLAB, we get some values of $a, b, c, \alpha$. As the Shilnikov sense Smale horseshoe chaos is predicted to occur in a range of $5>\alpha>0$, so we have (1).

$$
\begin{aligned}
& a=0.000000000000001 \text {, } \\
& \mathrm{b}=-0.000000000000023 \text {, } \\
& \mathrm{c}=0.000000000000000 \text {, } \\
& \alpha=0.279079600447614 \text {, } \\
& \left\{\begin{array}{l}
a_{1} \\
a_{2} \\
a_{3}
\end{array}\right\}=\left\{\begin{array}{c}
-2.771961941202775 \times 10^{-15} \\
6.271831842450346 \times 10^{-15} \\
-2.499869901247553 \times 10^{-15}
\end{array}\right\}, \\
& \left\{\begin{array}{l}
b_{1} \\
b_{2} \\
b_{3}
\end{array}\right\}=\left\{\begin{array}{c}
-7.071703786216559 \times 10^{-14} \\
7.169219264330171 \times 10^{-14} \\
-2.397515478113609 \times 10^{-14}
\end{array}\right\} \text {, } \\
& \left\{\begin{array}{l}
c_{1} \\
c_{2} \\
c_{3}
\end{array}\right\}=\left\{\begin{array}{c}
-2.676214696157709 \times 10^{-42} \\
4.186429392315434 \times 10^{-42} \\
-1.510214696157717 \times 10^{-42}
\end{array}\right\} \text {, }
\end{aligned}
$$




$$
\begin{aligned}
& \left\{\begin{array}{l}
\mathrm{a}_{1,0} \\
\mathrm{a}_{2,0} \\
\mathrm{a}_{0,1} \\
\mathrm{a}_{0,2} \\
\mathrm{a}_{1,1}
\end{array}\right\}=\left\{\begin{array}{c}
-1.713682144486563 \times 10^{-15}-7.362315823177537 \times 10^{-15_{\mathrm{i}}} \\
3.715338502258792 \times 10^{-16}+4.623761973321532 \times 10^{-16_{\mathrm{i}}} \\
-1.713682144486563 \times 10^{-15}+7.362315823177537 \times 10^{-15} \mathrm{i} \\
3.715338502258792 \times 10^{-16}-4.623761973321532 \times 10^{-16_{i}} \\
3.684296588521366 \times 10^{-15}
\end{array}\right\}, \\
& \left\{\begin{array}{l}
\mathrm{b}_{1,0} \\
\mathrm{~b}_{2,0} \\
\mathrm{~b}_{0,1} \\
\mathrm{~b}_{0,2} \\
\mathrm{~b}_{1,1}
\end{array}\right\}=\left\{\begin{array}{c}
-4.107317993814730 \times 10^{-15}+4.318473674050183 \times 10^{-15_{\mathrm{i}}} \\
-1.381097237208684 \times 10^{-16}-4.503096154769760 \times 10^{-16} \mathrm{i} \\
-4.107317993814730 \times 10^{-15}-4.318473674050183 \times 10^{-15} \mathrm{i} \\
-1.381097237208684 \times 10^{-16}+4.503096154769760 \times 10^{-16_{i}} \\
-1.450914456492880 \times 10^{-14} \\
c_{1,0} \\
c_{2,0} \\
c_{0,1} \\
c_{0,2} \\
c_{1,1}
\end{array}\right\}=\left\{\begin{array}{c}
-5.547570401811642 \times 10^{-41}-1.989647415734912 \times 10^{-41} \mathrm{i} \\
1.325916367551282 \times 10^{-41}+7.496684227115509 \times 10^{-43} \mathrm{i} \\
-5.547570401811642 \times 10^{-41}+1.989647415734912 \times 10^{-41} \mathrm{i} \\
1.325916367551282 \times 10^{-41}-7.496684227115509 \times 10^{-43} \mathrm{i} \\
8.443308068520719 \times 10^{-41}
\end{array}\right\},
\end{aligned}
$$

$$
\begin{aligned}
\chi^{[+]}(t)= & \left(-2.771961941202775 \times 10^{-15}\right) e^{-5 t}+\left(6.271831842450346 \times 10^{-15}\right) e^{-5 t \times 2} \\
& +\left(-2.499869901247553 \times 10^{-15}\right) e^{-5 t \times 3}
\end{aligned}
$$$$
y^{[+]}(t)=\left(-7.071703786216559 \times 10^{-14}\right) e^{-5 t}+\left(7.169219264330171 \times 10^{-14}\right) e^{-5 t \times 2}
$$$$
+\left(-2.397515478113609 \times 10^{-14}\right) e^{-5 t \times 3} \text {, }
$$

$$
\begin{aligned}
z^{[+]}(t)= & \left(-2.676214696157709 \times 10^{-42}\right) e^{-5 t}+\left(4.186429392315434 \times 10^{-42}\right) e^{-5 t \times 2} \\
& +\left(-1.510214696157717 \times 10^{-42}\right) e^{-5 t \times 3},
\end{aligned}
$$

where (4.12), (4.13), and (4.14) are one dimensional invariant manifold. And

$$
\begin{aligned}
x^{[-]}(\mathrm{t})= & \operatorname{Re}\left(\left(-1.713682144486563 \times 10^{-15}-7.362315823177537 \times 10^{-15} \mathrm{i}\right) \mathrm{e}^{(0.279079600447614-1.000000000000000 \mathrm{i}) \mathrm{t}}\right. \\
& +\left(3.715338502258792 \times 10^{-16}+4.623761973321532 \times 10^{-16} \mathrm{i}\right) \mathrm{e}^{(0.558159200895228-2.000000000000000 \mathrm{i}) \mathrm{t}} \\
& +\left(-1.713682144486563 \times 10^{-15}+7.362315823177537 \times 10^{-15} \mathrm{i}\right) \mathrm{e}^{(0.279079600447614+1.000000000000000 \mathrm{i}) \mathrm{t}} \\
& +\left(3.715338502258792 \times 10^{-16}-4.623761973321532 \times 10^{-16} \mathrm{i}\right) \mathrm{e}^{(0.558159200895228+2.000000000000000 \mathrm{i}) \mathrm{t}} \\
& \left.+\left(3.684296588521366 \times 10^{-15}\right) \mathrm{e}^{0.558159200895228 \mathrm{t}}\right), \\
\mathrm{y}^{[-]}(\mathrm{t})= & \operatorname{Re}\left(\left(-4.107317993814730 \times 10^{-15}+4.318473674050183 \times 10^{-15} \mathrm{i}\right) \mathrm{e}^{(0.279079600447614-1.000000000000000 \mathrm{i}) \mathrm{t}}\right. \\
& +\left(-1.381097237208684 \times 10^{-16}-4.503096154769760 \times 10^{-16} \mathrm{i}\right) \mathrm{e}^{(0.558159200895228-2.000000000000000 \mathrm{i}) \mathrm{t}} \\
& +\left(-4.107317993814730 \times 10^{-15}-4.318473674050183 \times 10^{-15} \mathrm{i}\right) \mathrm{e}^{(0.279079600447614+1.000000000000000 \mathrm{i}) \mathrm{t}} \\
& +\left(-1.381097237208684 \times 10^{-16}+4.503096154769760 \times 10^{-16} \mathrm{i}\right) \mathrm{e}^{(0.558159200895228+2.000000000000000 \mathrm{i}) \mathrm{t}} \\
& \left.+\left(-1.450914456492880 \times 10^{-14}\right) e^{0.558159200895228 \mathrm{t}}\right), \\
z^{[-]}(\mathrm{t})= & \operatorname{Re}\left(\left(-5.547570401811642 \times 10^{-41}-1.989647415734912 \times 10^{-41} \mathrm{i}\right) \mathrm{e}^{(0.279079600447614-1.000000000000000 \mathrm{i}) \mathrm{t}}\right. \\
& +\left(1.325916367551282 \times 10^{-41}+7.496684227115509 \times 10^{-43} \mathrm{i}\right) \mathrm{e}^{(0.558159200895228-2.000000000000000 \mathrm{i}) \mathrm{t}} \\
& +\left(-5.547570401811642 \times 10^{-41}+1.989647415734912 \times 10^{-41} \mathrm{i}\right) \mathrm{e}^{(0.279079600447614+1.000000000000000 \mathrm{i}) \mathrm{t}} \\
& +\left(1.325916367551282 \times 10^{-41}-7.496684227115509 \times 10^{-43} \mathrm{i}\right) e^{(0.558159200895228+2.000000000000000 \mathrm{i}) \mathrm{t}} \\
& \left.+\left(8.443308068520719 \times 10^{-41}\right) e^{0.558159200895228 \mathrm{t}}\right) .
\end{aligned}
$$

Above equations are two dimensional invariant manifold. 
This moment, the 3D phase diagram is shown in Figure 1 and the time series of trajectories $x(t), y(t)$, and $z(t)$ are shown in Figure 2. Then, by bringing $\alpha=0.279079600447614$ back to the practical system (3.3), we calculate the deletion error is 0.017096611495099 with least squares and the deletion error generated by $\left(x_{0}, y_{0}, z_{0}\right)$. When $\alpha=0.279079600447614$, analysis reveals that the maximum Lyapunov exponent $\left(l_{1}=0.280875\right)$ is greater than zero, then it meets the exponential requirement for chaotic attractor.

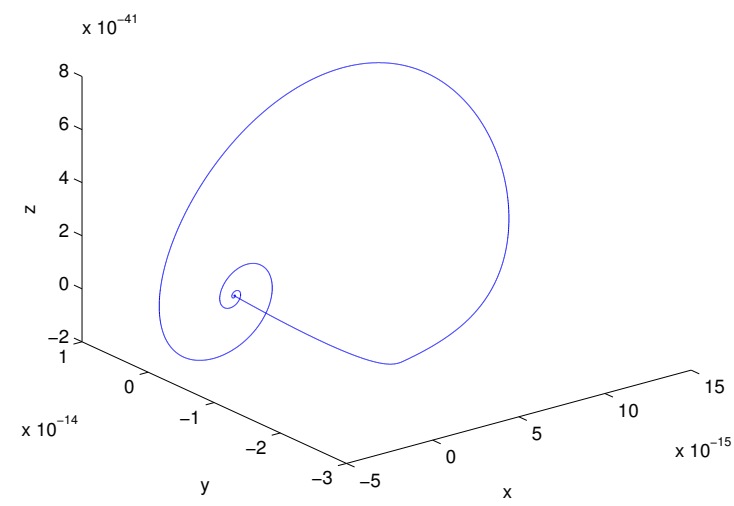

Figure 1: The 3D phase diagram of the model.
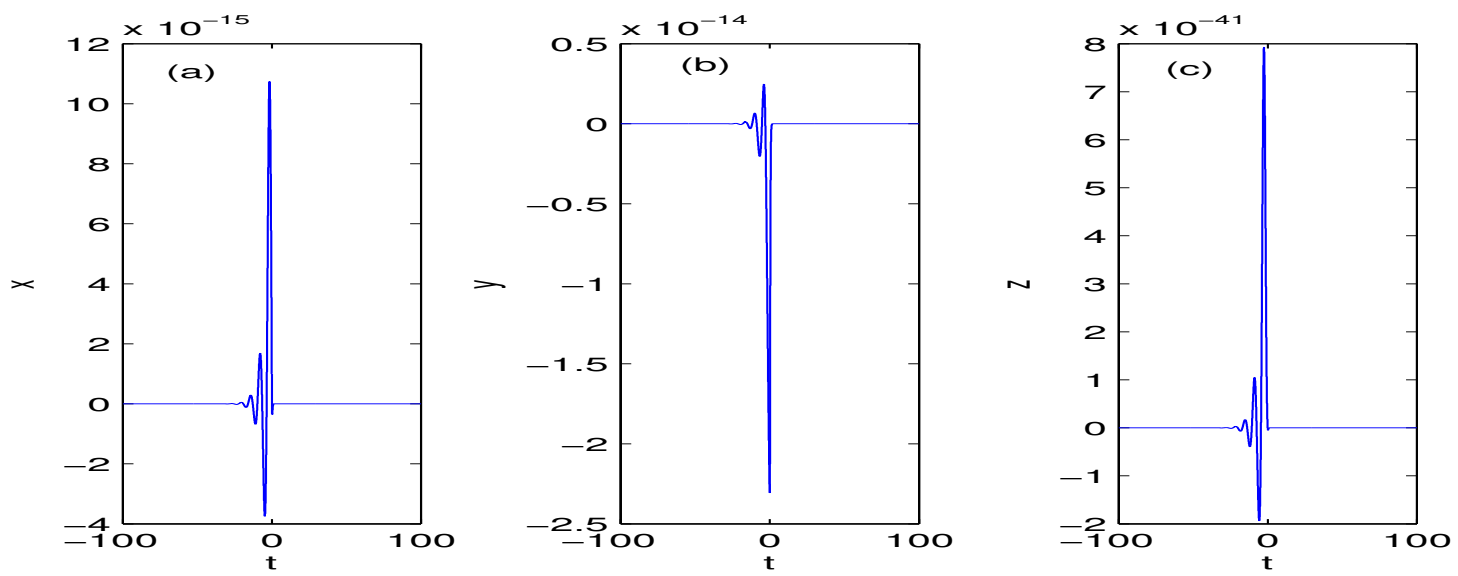

Figure 2: Time series of the trajectories of the model: (a) $x(t),(b) y(t)$, and (c) $z(t)$.

(2).

$$
\begin{aligned}
a & =-0.000000000000001, \\
b & =0.000000000000015, \\
c & =0.000000000000000, \\
\alpha & =0.128629219182314, \\
\left\{\begin{array}{l}
a_{1} \\
a_{2} \\
a_{3}
\end{array}\right\} & =\left\{\begin{array}{c}
7.501249025340232 \times 10^{-16} \\
-3.013112726986300 \times 10^{-15} \\
1.262987824452266 \times 10^{-15}
\end{array}\right\},
\end{aligned}
$$




$$
\begin{aligned}
& \left\{\begin{array}{l}
b_{1} \\
b_{2} \\
b_{3}
\end{array}\right\}=\left\{\begin{array}{c}
4.563572917579219 \times 10^{-14} \\
-4.597851452281091 \times 10^{-14} \\
1.534278534701872 \times 10^{-14}
\end{array}\right\}, \\
& \left\{\begin{array}{l}
c_{1} \\
c_{2} \\
c_{3}
\end{array}\right\}=\left\{\begin{array}{c}
7.177796569898546 \times 10^{-43} \\
-1.119159313979713 \times 10^{-42} \\
4.013796569898567 \times 10^{-43}
\end{array}\right\}, \\
& \left\{\begin{array}{l}
\mathrm{a}_{1,0} \\
\mathrm{a}_{2,0} \\
\mathrm{a}_{0,1} \\
\mathrm{a}_{0,2} \\
\mathrm{a}_{1,1}
\end{array}\right\}=\left\{\begin{array}{c}
3.904148455119211 \times 10^{-16}+4.533912565983060 \times 10^{-15_{\mathrm{i}}} \\
-1.028534829823685 \times 10^{-16}-3.186109474304711 \times 10^{-16} \mathrm{i} \\
3.904148455119211 \times 10^{-16}-4.533912565983060 \times 10^{-15_{i}} \\
-1.028534829823685 \times 10^{-16}+3.186109474304711 \times 10^{-16} \mathrm{i} \\
-1.575122725059106 \times 10^{-15}
\end{array}\right\}, \\
& \left\{\begin{array}{l}
\mathrm{b}_{1,0} \\
\mathrm{~b}_{2,0} \\
\mathrm{~b}_{0,1} \\
\mathrm{~b}_{0,2} \\
\mathrm{~b}_{1,1}
\end{array}\right\}=\left\{\begin{array}{c}
2.827844737584515 \times 10^{-15}-1.106610813994100 \times 10^{-15_{\mathrm{i}}} \\
-5.004118701902976 \times 10^{-17}+1.366377793786894 \times 10^{-16_{\mathrm{i}}} \\
2.827844737584515 \times 10^{-15}+1.106610813994100 \times 10^{-15_{i}} \\
-5.004118701902976 \times 10^{-17}-1.366377793786894 \times 10^{-16_{i}} \\
9.444392898869032 \times 10^{-15}
\end{array}\right\}, \\
& \left\{\begin{array}{l}
c_{1,0} \\
c_{2,0} \\
c_{0,1} \\
c_{0,2} \\
c_{1,1}
\end{array}\right\}=\left\{\begin{array}{c}
1.376061727611506 \times 10^{-41}+4.310032277211528 \times 10^{-42} \mathrm{i} \\
-3.101060515260080 \times 10^{-42}-8.745074107590937 \times 10^{-43} \mathrm{i} \\
1.376061727611506 \times 10^{-41}-4.310032277211528 \times 10^{-42} \mathrm{i} \\
-3.101060515260080 \times 10^{-42}+8.745074107590937 \times 10^{-43} \mathrm{i} \\
-2.131911352170996 \times 10^{-41}
\end{array}\right\},
\end{aligned}
$$

$$
\begin{aligned}
\chi^{[+]}(t)= & \left(7.501249025340232 \times 10^{-16}\right) e^{-5 t}+\left(-3.013112726986300 \times 10^{-15}\right) e^{-5 t \times 2} \\
& +\left(1.262987824452266 \times 10^{-15}\right) e^{-5 t \times 3}
\end{aligned}
$$$$
y^{[+]}(t)=\left(4.563572917579219 \times 10^{-14}\right) e^{-5 t}+\left(-4.597851452281091 \times 10^{-14}\right) e^{-5 t \times 2}
$$$$
+\left(1.534278534701872 \times 10^{-14}\right) e^{-5 t \times 3},
$$$$
z^{[+]}(t)=\left(7.177796569898546 \times 10^{-43}\right) e^{-5 t}+\left(-1.119159313979713 \times 10^{-42}\right) e^{-5 t \times 2}
$$$$
+\left(4.013796569898567 \times 10^{-43}\right) e^{-5 t \times 3} \text {, }
$$

where (4.15), (4.16), and (4.17) are the one dimensional invariant manifold. And

$$
\begin{aligned}
x^{[-]}(\mathrm{t})= & \operatorname{Re}\left(\left(3.904148455119211 \times 10^{-16}+4.533912565983060 \times 10^{-15} \mathrm{i}\right) e^{(0.128629219182314-1.000000000000000 \mathrm{i}) \mathrm{t}}\right. \\
& +\left(-1.028534829823685 \times 10^{-16}-3.186109474304711 \times 10-16 \mathrm{i}\right) \mathrm{e}^{(0.257258438364628-2.000000000000000 \mathrm{i}) \mathrm{t}} \\
& +\left(3.904148455119211 \times 10^{-16}-4.533912565983060 \times 10^{-15} \mathrm{i}\right) \mathrm{e}^{(0.128629219182314+1.000000000000000 \mathrm{i}) \mathrm{t}} \\
& +\left(-1.028534829823685 \times 10^{-16}+3.186109474304711 \times 10^{-16} \mathrm{i}\right) e^{(0.257258438364628+2.000000000000000 \mathrm{i}) \mathrm{t}} \\
& \left.+\left(-1.575122725059106 \times 10^{-15}\right) e^{0.257258438364628 \mathrm{t}}\right), \\
\mathrm{y}^{[-]}(\mathrm{t})= & \operatorname{Re}\left(\left(2.827844737584515 \times 10^{-15}-1.106610813994100 \times 10^{-15} \mathrm{i}\right) \mathrm{e}^{(0.128629219182314-1.000000000000000 \mathrm{i}) \mathrm{t}}\right. \\
& +\left(-5.004118701902976 \times 10^{-17}+1.366377793786894 \times 10^{-16} \mathrm{i}\right) \mathrm{e}^{(0.257258438364628-2.000000000000000 \mathrm{i}) \mathrm{t}} \\
& +\left(2.827844737584515 \times 10^{-15}+1.106610813994100 \times 10^{-15} \mathrm{i}\right) e^{(0.128629219182314+1.000000000000000 \mathrm{i}) \mathrm{t}} \\
& +\left(-5.004118701902976 \times 10^{-17}-1.366377793786894 \times 10^{-16} \mathrm{i}\right) e^{(0.257258438364628+2.000000000000000 \mathrm{i}) \mathrm{t}} \\
& \left.+\left(9.444392898869032 \times 10^{-15}\right) e^{0.257258438364628 \mathrm{t}}\right), \\
z^{[-]}(\mathrm{t})= & \operatorname{Re}\left(\left(1.376061727611506 \times 10^{-41}+4.310032277211528 \times 10^{-42} \mathrm{i}\right) e^{(0.128629219182314-1.000000000000000 \mathrm{i}) \mathrm{t}}\right.
\end{aligned}
$$




$$
\begin{aligned}
& +\left(-3.101060515260080 \times 10^{-42}-8.745074107590937 \times 10^{-43} \mathrm{i}\right) \mathrm{e}^{(0.257258438364628-2.000000000000000 \mathrm{i}) \mathrm{t}} \\
& +\left(1.376061727611506 \times 10^{-41}-4.310032277211528 \times 10^{-42} \mathrm{i}\right) \mathrm{e}^{(0.128629219182314+1.000000000000000 \mathrm{i}) \mathrm{t}} \\
& +\left(-3.101060515260080 \times 10^{-42}+8.745074107590937 \times 10^{-43} \mathrm{i}\right) \mathrm{e}^{(0.257258438364628+2.000000000000000 \mathrm{i}) \mathrm{t}} \\
& \left.+\left(-2.131911352170996 \times 10^{-41}\right) e^{0.257258438364628 \mathrm{t}}\right) .
\end{aligned}
$$

Above equations are the two dimensional invariant manifold.

This moment, the 3D phase diagram is shown in Figure 3 and the time series of trajectories $x(t), y(t)$, and $z(t)$ are shown in Figure 4. Then, by bringing $\alpha=0.128629219182314$ back to the practical system (3.3) we calculate the deletion error is 0.046683594397385 with least squares and the deletion error generated by $\left(x_{0}, y_{0}, z_{0}\right)$. When $\alpha=0.128629219182314$, analysis reveals that the maximum Lyapunov exponent $\left(l_{1}=0.12\right)$ is greater than zero, then it meets the exponential requirement for chaotic attractor.

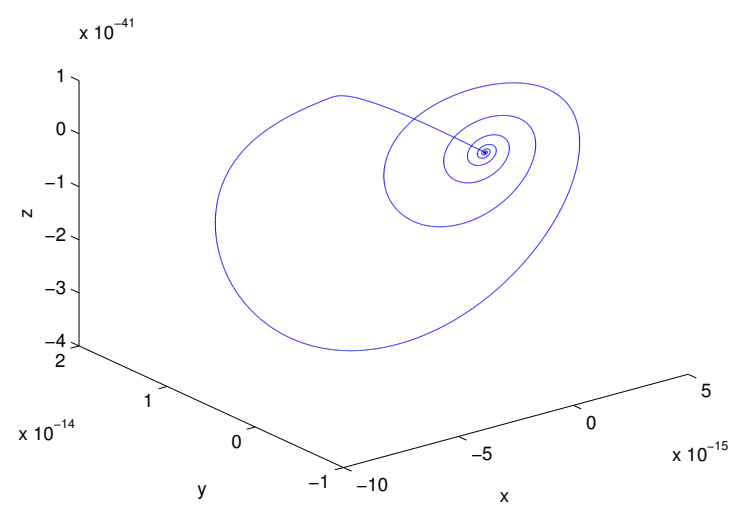

Figure 3: The 3D phase diagram of the model.
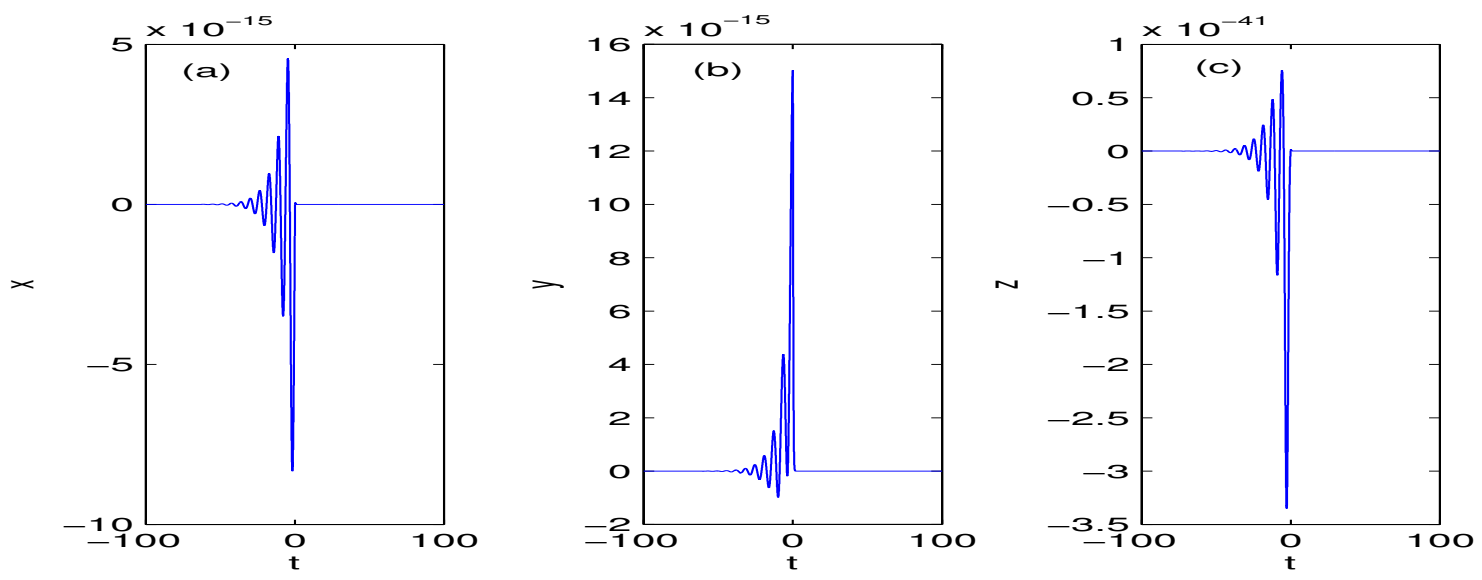

Figure 4: Time series of the trajectories of the model: (a) $x(t),(b) y(t)$, and (c) $z(t)$.

From Figure 1 and Figure 3, we can see that the focus becomes more and more weak as $\alpha \rightarrow 0$. It is easy to prove this conclusion from a mathematical point of view.

When $\alpha \approx 0.279079600447614$ or $\alpha \approx 0.128629219182314$, the above two expressions are close to homoclinic orbit of the system (3.3) very much. Moreover, according to continuity of solution for initial value and parameter, we know when $\alpha \approx 0.279079600447614$ or $\alpha \approx 0.128629219182314$, the system 
is likely to exist the homoclinic orbit. Then, by the Shilnikov theorem, the system is likely to exhibit horseshoe chaos.

\section{Conclusion}

In this article, the homoclinic orbit of the system is constructed by the series expression of the stable and unstable manifold and power series expansion in the neighborhood of $t=0$. The Shilnikov sense chaotic motion is predicted by the Shilnikov theorem. In this paper, we just find an orbit approximates the homoclinic orbit. If we want to get the practical homoclinic orbit precisely, we have more works to do and more challenges to face.

The strategy presented in this paper is generally applied and accepted. Hence, it would be possible to apply the presented method to research other 3D systems with more complicated nonlinearity, which will be a topic for the further study.

\section{References}

[1] E. Emaci, A. F. Vakakis, I. V. Andrianov, Y. Mikhlin, Study of two-Dimensional axisymmetric breathers using Padé approximants, Nonlinear Dynam., 13 (1997), 327-338. 1

[2] J. J. Feng, Q. C. Zhang, W. Wang, The construction of homoclinic and heteroclinic orbitals in asymmetric strongly nonlinear systems based on the Pade approximant, Chinese Physics B, 20 (2011), 19-29. 1

[3] J. J. Feng, Q. C. Zhang, W. Wang, Chaos of several typical asymmetric systems, Chaos Solitons and Fractals, 45 (2012), 950-958. 1

[4] J. J. Feng, Q. C. Zhang, W. Wang, S. Y. Hao, Homoclinic orbits in three-dimensional Shilnikov-type chaotic systems, Chinese Physics B, 22 (2013), 312-323. 1

[5] Y. H. Li, S. M. Zhu, n-Dimensional stable and unstable manifolds of hyperbolic singular point, Chaos Solitons Fractals, 29 (2006), 1155-1164. 1, 4

[6] G. V. Manucharyan, Y. V. Mikhlin, The construction of homo-and heteroclinic orbits in non-linear systems, J. Appl. Math. Mech., 69 (2005), 39-48. 1

[7] Y. V. Mikhlin, Matching of local expansions in the theory of non-linear vibrations, J. Sound Vibration, 182 (1995), 577588. 1

[8] Y. V. Mikhlin, Analytical construction of homoclinic orbits of two-and three-dimensional Dynamical Systems, J. Sound Vibration, 230 (2000), 971-983. 1

[9] A. F. Vakakis, M. F. A. Azeez, Analytic approximation of the homoclinic orbits of the lorenz system at $\sigma=10, \mathrm{~b}=8 / 3$ and $\rho=13.926 \ldots$, Nonlinear Dynam., 15 (1998), 245-257. 1

[10] W. Wang, Q. C. Zhang, R. L. Tian, Shilnikov sense chaos in a simple three dimensional system, Chinese Physics B, 19 (2010), 207-216. 1, 2.1, 4 